\title{
I.N. Petrov, The Development of the Bulgarian Literary Language. From Incunabula to First Grammars. Late Fifteenth-Early Seventeenth Century, Lexington Books, Lanham 2021, pp. 190.
}

Lo studio Od inkunabutów do pierwszych gramatyk. Konteksty rozwoju butgarskiego jezyka literackiego (koniec XV-poczatek XVII wiek), uscito per i tipi della Łódź University Press (20I5), è stato recentemente pubblicato in versione inglese nella collana "Studies in Slavic, Baltic and Eastern European Languages and Cultures", diretta da Andrii Danylenko.

Questo lavoro, già positivamente recensito nella versione polacca, offre un ampio panorama della stampa in cirillico di tradizione ortodossa dalle origini fino all'inizio del Xvir secolo, illustrando un momento fondamentale della storia della lingua bulgara. L'autore, Ivan N. Petrov, direttore del Dipartimento di Filologia slava dell'Università di Eódź e del Centro di ricerca Ceraneum, descrive la produzione libraria slava meridionale in chiave multidisciplinare, spaziando dalla linguistica alla storia del libro a stampa in una prospettiva storico-culturale capace di superare ogni visione angustamente nazionale.

Il primo capitolo contiene una riflessione sulle diverse definizioni di 'slavo ecclesiastico' sul piano teorico e metodologico, considerando in particolare i problemi di periodizzazione. Questo panorama è assai utile per orientarsi fra le diverse posizioni. Fa piacere riconoscere in questa riflessione concetti a noi familiari, come quello di Slavia orthodoxa, con riferimenti agli studi di R. Picchio. Per le definizioni si citano in particolare N. Tolstoj e E. Vereščagin, mentre per il suo uso nella letteratura scientifica si considerano soprattutto le ricerche di A. Naumow. Lo studioso si concentra specialmente sul periodo più recente dello slavo ecclesiastico, il 'nuovo slavo ecclesiastico' (abbreviato in inglese in NCS), in un contesto che vide dominare la produzione tipografica proveniente dall'impero russo, legata alla riforma del patriarca Nikon, e consolidare le sue forme nella cosiddetta Bibbia di Elisabetta (San Pietroburgo 175I).

Con chiarezza lo studioso evidenzia le questioni chiave che definiscono "the position and historical role of NCS in the Slavia Orthodoxa linguistic system":

I. l'ascesa e lo sviluppo della stampa cirillica nelle aree slave meridionali e orientali;

2. l'attività revisionale e redazionale di Massimo il Greco nel xvi secolo;

3. lo sviluppo del pensiero grammaticale tra gli slavi ortodossi e i conseguenti processi di codificazione: l'apparizione dei primi abbecedari, lessici e grammatiche;

4. la riforma della Chiesa del Xvir secolo in Russia e il suo impatto sulla lingua; 
5. lo sforzo di stabilizzare i testi canonici e di unificare il testo della Bibbia in Cs e NCs; la nascita dei primi manoscritti e codici stampati contenenti tutti i libri della Bibbia;

6. l'istituzione di regolamenti che determinano gli standard di scrittura dei testi nel NCs, introdotti in Russia nel XVIII secolo, e il loro aggiornamento fino ai giorni nostri;

7. la correlazione tra l'origine locale (dialettale) e la dimensione sovranazionale del NCs (con un richiamo alla somiglianza funzionale e tipologica con l'antico slavo ecclesiastico, da cui il NCS deriva).

Già da questo prospetto emerge il processo di standardizzazione promosso dalla traduzione e revisione dei testi biblici, ma allo stesso tempo il ruolo che ha assunto l'opera di Massimo il Greco. La sua azione a nostro parere andrebbe considerata non soltanto in relazione alla sua personale attività di revisione e redazione, ma anche in rapporto allo sviluppo del pensiero grammaticale e della sua codificazione.

Dovendo riconoscere che l'attenzione degli studiosi si è concentrata soprattutto sull'antico slavo ecclesiastico, Petrov cerca di ricostruire un quadro complessivo delle ricerche a disposizione citando, per la ricostruzione dei modelli teorici e metodologici di descrizione dello slavo ecclesiastico, oltre ai sunnominati Picchio e Naumow, V. Mošin, R. Mathiesen, H. Birnbaum, D.S. Worth e N. Trunte. A Picchio si fa riferimento in particolare per il concetto di 'dignità' e di 'norma', nonché per la classica raccolta di studi sulla 'questione della lingua' da lui curata. Si offre ugualmente spazio allo stato degli studi sull'“influenza dello slavo ecclesiastico sul bulgaro", in cui domina l'idea di considerare l'epoca delle prime stampe il periodo finale della lunga "fase pre-nazionale della storia del bulgaro", caratterizzata dalla diffusione della tradizione libraria slava orientale e russa e dalle relazioni culturali con l'impero russo.

Nel secondo capitolo Petrov riflette sulla classificazione e periodizzazione della produzione libraria più antica, considerando le differenti definizioni terminologiche (incunaboli, paleotipi), le diverse fasi, l'uso degli alfabeti, e facendo riferimento ai cataloghi disponibili di stampe cirilliche, a cominciare dalle pubblicazioni di E. Nemirovskij. Per lo studio di questa produzione libraria, a suo parere, è fondamentale considerare i metatesti: dediche, prefazioni, postfazioni, preghiere degli stampatori, colofoni.

Nel terzo capitolo, che costituisce il contributo più originale del volume, si descrivono, con interessanti osservazioni sulla letteratura critica, i libri a stampa, raggruppati secondo queste diverse categorie: gli incunaboli glagolitici e cirillici, le edizioni rumeno-bulgare dell'inizio del XVI secolo, le edizioni veneziane del XVI-XVII secolo, i paleotipi serbi del XVI sec., le edizioni rumeno-bulgare della metà del XVI sec., i paleotipi slavi orientali. Il lodevole scopo è "an attempt to organize the dispersed information, observations and facts, to arrange them into a factual and methodological whole, thus making it possible to uncover areas that are still debatable or unknown". Va sottolineato in generale che si tratta soprattutto di libri di uso liturgico, dai vangeli al salterio, ma anche di libri per la preghiera personale (soprattutto da viaggio). Fra i testi più importanti si può citare il Vangelo di Târgoviște (I5I2), il primo vangelo a stampa della Slavia Orthodoxa, mentre fra gli stampatori si pone attenzione, per esempio, al diacono Coresi (I557-1583) e a F. Skaryna. Fra i luoghi di stampa, non sempre definibili con certezza, emerge Vilnius.

Nelle sue conclusioni l'autore invita a considerare in una nuova prospettiva il ruolo dello slavo ecclesiastico per la lingua bulgara, non tanto sul piano del contatto linguistico, quanto piuttosto considerando in chiave diacronica i processi interni di sviluppo, che sono comuni all'intera area 
slava meridionale. Questo, tuttavia, comporta uno studio parallelo della codificazione dello slavo ecclesiastico nella riflessione linguistica e grammaticale che esula dal volume proposto.

La chiarezza dell'esposizione, l'attenzione ai problemi metodologici e alle definizioni, la presentazione delle diverse tesi, lo sforzo di bilanciare l'analisi concreta dei dati con una equilibrata riflessione teorica, come pure l'imponente Bibliografia, ne fanno uno strumento fondamentale per le future ricerche.

Marcello Garzaniti 